\title{
Photographic Capture-Recapture for Free-Roaming Dog Population Estimation: Is It Possible to Optimize the Dog Photo-Identification?
}

\author{
Shimozako HJ ${ }^{1,2}$, Euro de Barros Couto Junior ${ }^{3}$, Paulo Sergio Panse Silveira ${ }^{1}$ and Eduardo Massad ${ }^{1,4,5}$ \\ ${ }^{1}$ School of Medicine and LIM 01-HCFM, University of São Paulo, São Paulo SP, Brazil \\ ${ }^{2}$ Faculty of Philosophy Sciences and Literature of Ribeirão Preto and RIDC NeuroMat, University of São Paulo, São Paulo SP, Brazil \\ ${ }^{3}$ São Paulo Municipal Government - Health Department of Public Agents - Patriarca Square, São Paulo SP, Brazil \\ ${ }^{4}$ School of Applied Mathematics, Getulio Vargas Foundation, Rio de Janeiro RJ, Brazil \\ ${ }^{5}$ London School of Hygiene and Tropical Medicine, University of London, London, UK
}

Submission: February 13, 2018; Published: February 28, 2018

*Corresponding author: Helio Junji Shimozako, Faculty of Philosophy Sciences and Literature of Ribeirao Preto, University of São Paulo, Ribeirão Preto SP, Brazil, Tel: +55 11 30617435; Email: hjunji21@usp.br

\begin{abstract}
It is important to estimate the population of free-roaming dogs because this information helps with planning and developing appropriate dog population control strategies as well as with epidemiological surveillance services. The photographic capture-recapture method has been used to estimate animal populations, including free-roaming dogs. This method consider the natural marks of phenotypical details that make a dog distinguishable from each other. However, the risk of misidentification is one of the limitations of the photographic capture-recapture method. In addition, the interference of the inhabitants of the study area is also other point to be considered. In this paper, it is discussed some current implications regarding to the application of photographic capture-recapture method to estimate free-roaming dog population. In addition, it is presented some possible directions to reduce the impact of such limitations.
\end{abstract}

Keywords : Photographic capture-recapture; Free-roaming dog; Dog population estimation; Veterinary public health

\section{Introduction}

Dogs are undoubtedly one of the most popular domestic animals in the world. Although most dogs are considered domiciliary and restricted animals, there are some of them that freely access the streets (they are known as free-roaming dogs). In the case of free-roaming dog population, there are some estimation techniques (or methods) that can be used to estimate the population abundance (i.e., there are indirect methods, surveys based on counts, lines, transects or points, radio telemetry and plenty of other different forms of captureand-recapture methods) [1]. The capture-recapture method has been used for estimating wild animal populations and has been adapted for free-roaming dogs [1-4]. This method provides estimates of dog population sizes that can be important for the planning and development of appropriate control strategies, and to aid surveillance services [1-7]. Additionally, all descriptive data regarding the free-roaming dog population may generate information on dog control issues. This collected information can be utilized to inform dog control strategies at the local authority and to complement existing responsible dog ownership education programs [8]. Therefore, obtaining precise data of free-roaming dog population is interesting for preventive approach of veterinary public health. In this paper, it is discussed some limitations observed on photographic capture-recapture method when applied to estimate free-roaming dog population size. In addition, some directions and ideas to minimize such difficulties are pointed. All methods based on capture-recapture require a marking technique in order to identify each individual animal. Marking by photo-identification is an alternative that avoids physical contact between the researchers and the animals of unknown origin $[2,3,7,9]$. In addition, photo-identification avoids some of the problems traditionally associated with applying physical tags. For instance, natural marks cannot be shed, removed or fouled, and, depending on field practices, can also minimize the risk of confounding stress or behavioral issues [10].

The photo-identification process should consider that there is not misidentification of the animals [10]. However, some works have considered the possibility of misidentification [1114]. Moreover, one of the major criticisms of photo-identification studies is their perceived subjectivity, particularly in the initial identification and in the subsequent photo-matching [10]. The misidentification of photographs has been studied in the 


\section{Biostatistics and Biometrics Open Access Journal}

case of captive and wildlife populations. As illustration, some works have reported the use of digital technology to match photographs [15-18], the use of a biomolecular gold standard to compare with the photographic basis [19], and the description of methods of correction that can be used for making population estimations [14]. As alternative examples, the questions related to misidentification have also been studied by Dala Corte et al. [11] for Rineloricaria aequalicuspis Reis \& Cardoso (Siluriformes: Loricariidae), where the authors demonstrated the potential use of the photo-identification technique for this species. Marshall and Pierce [10] performed a review on using photo-identification for sharks and rays and mentioned that it is necessary to establish whether the focal species possesses sufficiently distinctive patterns or scars and, if so, where these identifiable marks occur. In other words, a standardized area or areas should be chosen for matching individuals between the two collection events.

On the one hand, captive and wildlife animals usually present a standard phenotype that allows the search for natural marks. For example, sharks and rays can be distinguished by physical characteristics of their fins [10]. On the other hand, few studies about the dog photo-identification have been available. A study conducted by Shimozako \& Couto Junior [3] developed a research in which had estimated the probabilities of photo-identification considering domestic dogs; proposed a correction method to compensate for the misidentification of photographed dogs; and demonstrated the feasibility and practicality of this calibration method in a real case study that estimates the free-roaming dog population. Although this work has been an interesting initiative in a novel question concerning dog population studies, a set of criticism arose about it. Firstly, a dog population study is commonly performed in an urban area and may require cooperation among researchers, institutions and public policy authorities. However, it is very usual to observe limitations related to material and human resources or conflict of schedule. In addition, the study area may not properly fit to method assumptions or research conditions. Several reasons may explain a possibility of rejection of such area, as lack of safety or geographical unavailability. Although those conditions can become the performing of the research unfavorable, sometimes such situations are the best one obtained. As result, a combination of factors related to availability of resources and general characteristics of the study area may interfere in the feasibility of the research, as well in the quality of the results.

Once defined the study area and the logistic of collecting data (the pictures of dogs), the challenge is to apply the photographic capture-recapture method. Considering a wildlife or captive animal studies, it is well know that the presence of the researcher can potentially interfere on the behavior of the individuals $[10,20]$. In the case of free-roaming dog population, it is usual to find people that support those dogs with food, water and shelter $[2,3,5,6,9]$. Therefore, different from a wildlife or captive population, the dog population receives support from natural and human interferences. Such human interference can also offer protection to those animals. As consequence, because of the necessity to search for free-roaming dog (by walking or car, for example), the inhabitants may bring the dogs inside their houses during the research period (possibly, they believe the researchers can represent the dog remotion service). This fact was observed by Shimozako \& Couto Junior [3] in their research. Other point to be considered is the support team during the search for dog's pictures. Often, the members of such team has a specific time to work in the project and it is not able for them to cooperate more than this time. This is a serious problem, in particular considering a data collecting or sampling. The limitation of work time may influence both in the studied area to be covered and in the quality of data (or pictures). Again, the work developed by Shimozako \& Couto Junior [3] had been supported by their research institution and the members of this support team had a specific time to aid them on the data collecting. In addition, since the round-trip from the institution to the study area spent a total of 4 hours, this time had to be discounted to the available work time.

Finally, we mention again the question about the misidentification. After briefly explain about the limitations and intrinsic challenge considering photographic capturerecapture method to estimate free-roaming dog population, we can discuss about the possibility of optimize the photoidentification for dogs. Since there are researches to improve the accuracy of photo-identification of some wildlife and captive populations [10-19], what about dog population? At the best of our knowledge, Shimozako \& Couto Junior [3] is the only study that evaluated dog photo-identification. This study not only described some results due to the limitations and challenges of the application of photographic capture-recapture in urban areas, but also indicated how difficult is to find a phenotypical pattern to identify a dog. The dog has some particularities that make the optimization of photo-identification as a big challenge. This species presents several breeds, with different sizes and kind of fur. Even though it seems that this variability points to certainty of distinguishable dogs, how many dogs are necessary to estimate a probability of identification? How should we quantify a phenotype? How should we take the pictures? Here, "probability" should be calculated as the "correctly identified/ total of possibilities". But, other question arises here: how many attempts is it necessary? Note that it is necessary to estimate a dog sample size to obtain the pictures. Now, it is questioned how many attempts (observations) should be done in order to estimate how probable to identify a dog using its pictures is.

An interesting point need to be highlighted here. It is necessary to find an "equilibrium point" between the scientifically suitable and methodologically feasible. Shimozako \& Couto Junior [3] had accessed to Zoonosis Control Centre (ZCC) of São Paulo City and they were able to obtain pictures of captive dogs from adoption department. However, few dogs were weekly available to serve as "models". Since the research 
project had a limited time and the amount of dogs was low, the amount of model-dogs were also limited. In a statistical point of view, the problem of sample size calculation has dozens of formulas. Often, there are several ways to solve a given problem. According to Shimozako \& Couto Junior [3], it had not been adopted a previous calculation about how many dogs would be necessary for evaluating photo-identification, because there was no information (at the beginning of the experiment) about how many free-roaming dogs existed in the investigated region [21]. In those cases, a sequential observational procedure is commonly used to estimate the quantity of available elements. It is common to divide experiments like this in two or more strata: the first stratum can produce some previous results to the second one [22]. In this case, the amount of photos of the first strata has been used in the second strata, where we considered a mathematical combination of photos, forming pairs. Combinations of elements are a quite good method for optimization, i.e., for finding the most interesting solution to consider all possibilities of formed pairs [23].

Parametric procedures (based on normality) are often usable to calculate sample sizes, but in some cases, non-parametric methods are necessary. Non-parametric methods, based on the frequency of appearance of each investigated element, are simpler and prevent super-estimations. In general, it is preferable to avoid a super-estimated result in terms of sample size estimation [24]. Enumeration does not end with arithmetic: there is quite a bit more to it [25]. The problem to be solved does not belong to 'routine' proceedings. We should develop an ability to decompose the problem into such combinatorial and statistical problems [25]. Working with a finite population almost always consists of a pre-existent problem: it is not possible to know how to estimate the size of this population, and we must call upon to a secure mathematical technique to absorb the necessity of the needed calculation.

\section{Conclusion}

In the context of photographic capture-recapture, improving the accuracy of photo-identification is crucial for acquisition of better results. Although misidentification has been studied in wildlife and captive animals, few about it has been studied considering dog population. The misidentification is directly influenced by phenotypical characteristics of the dog, and it is indirectly dependent of human factors and research resources availability. However, the design of a proper evaluation study of dog photo-identification is an important challenge, due to the indeterminacy of sampling method for the amount dogs to be considered as phenotypical models and for the number of visits to perform the data collecting (that is, the search for freeroaming dogs). According to our expertise, to perform a study of photographic capture-recapture method is hard not only due to the methodological execution, but also because of external interferences on the study development. The dog population receives the interference from natural factors, but also from human community. This extra interference on dog population may also cause bias in the free-roaming dog population estimation.

Combinatorial methods used with some non-parametric techniques can produce good results in terms of sample size and population size calculations. Useful and traditional formulas for estimating the size of a population work better with frequency-based formulas of non-parametric methods for its determination. The combinatorial mathematical method used in the work performed by Shimozako \& Couto Junior [3] was one of the most simpler and regular methods for a good estimation.

\section{References}

1. Belo VS, Werneck GL, da Silva ES, Barbosa SB, Struchiner CJ (2015) Population Estimation Methods for Free-Ranging Dogs: A Systematic Review. PLoS ONE 10(12): e0144830.

2. Beck AM (1973) The ecology of stray dogs- A study of free-ranging urban animals. Purde University Press, West Lafayette, Indiana, USA, pp 116.

3. Shimozako HJ, Couto Junior EB (2010) Photographic capture-recapture for estimation of stray dog population: A methodological evaluation and an ecological study. VDM Verlag Dr. Müller, Saarbrücken, Sarre, Germany, pp. 92.

4. Wongphruksasoong V, Santayakorn S, Sitthi W, Ardkham B, Pisek S, et al. (2016) Census versus Capture-recapture Method to Estimate Dog Population in Lumlukka District, Pathum Thani Province, Thailand, 2010. OSIR Journal 9(1): 15-20.

5. Beck AM (1975) The Public Health Implications of Urban Dogs. Am J of Public Health 65(12): 1315-1318.

6. Wandeler AI, Matter HC, Kappeler A, Budde A (1993) The ecology of dogs and canine rabies: a selective review. Rev Sci Tech 12(1): 51-71.

7. Thrusfield M (2007) Veterinary Epidemiology ( $3^{\text {th }}$ edn), Blackwell Science, Oxford, Oxfordshire, UK, pp. 624.

8. O'Sullivan EN, Hanlon AJ (2012) A review of official data obtained from dog control records generated by the dog control service of county cork, Ireland during 2007. Ir Vet J 65(1): 10.

9. Bögel K, Frucht K, Drysdale G, Remfry J, World Health Organization (WHO) - Veterinary Public Health Unit, World Society for the Protection of Animals (WSAP) (1990) Guidelines for dog population management. WHO, Geneva, Switzerland.

10. Marshall AD, Pierce SJ (2012) The use and abuse of photographic identification in sharks and rays. J Fish Biol 80(5) 1361-1379.

11. Corte DRB, Moschetta JB, Becker FG (2016) Photo-identification as a technique for recognition of individual fish: a test with the freshwater armored catfish Rineloricaria aequalicuspis Reis \& Cardoso, 2001 (Siluriformes: Loricariidae). Neotrop Ichthyol 14(1): e150074.

12. Lima M de L (2016) Photography and memory in Information Science. Scire 22(1): 35-43.

13. Patton F, Jones M (2008) Error that occur when using photoidentification to identify individual black rhinos. Pachyderm 44: 35-44

14. Yoshizaki J, Pollock KH, Cavell B (2009) Modeling misidentification errors in capture-recapture studies using photographic identification of evolving marks. Ecology 90(1): 3-9.

15. Ardovini A, Cinque L, Sangineto E (2008) Identifying elephant photos by multi-curve matching. Pattern Recognition 41: 1867-1877.

16. Hastings KK, Hiby LA, Small RJ (2008) Evaluation of a computerassisted photograph-matching system to monitor naturally marked harbor seals at Tugidak Island, Alaska. J Mammal 89: 1201-1211. 
17. Reisser J, Proietti M, Kinas P, Sazima I (2008) Photographic identification of sea turtles: method description and validation, with an estimation of tag loss. Endang Species Res. 5: 73-82.

18. Jean C, Ciccione S, Talma E, Ballorain K, Bourjea J (2010) Photoidentification method for green and hawksbill turtles - First results from Reunion. Indian Ocean Turtle News. 11: 8-13.

19. Frasier TR, Hamilton PK, Brown MW, Kraus SD, White BN (2009) Sources and rates of errors in methods of individual identification for north Atlantic right whales. J Mammal 90: 1246-1255.

20. Amstrup SC, McDonald TL, Manly BFJ (2005) Handbook of capturerecapture analysis. Princeton University Press, Princeton, New Jersey, USA, pp. 336.

21. Pollock KH, Otto MC (1983) Robust estimation of population size in closed animal populations from capture-recapture experiments. Biometrics 39(4): 1035-1049.
22. Cochran WG (1986) Sampling techniques ( $3^{\text {rd }}$ edn). John Wiley and Sons, Toronto, Canada, pp. 428.

23. Riordan J (2002) An Introduction to Combinatorial Analysis - Dover Books on Mathematics. Dover Publications, Mineola, New York, USA, pp. 256.

24. Peres C de A, Saldiva CD (1982) Experiment Planning (only in Portuguese). V National Symposium on Probability and Statistics. IMEUSP, São Paulo, São Paulo, Brazil, pp. 98.

25. Grimaldi RP (1985) Discrete and Combinatorial Mathematics - an applied introduction. Addison-Wesley Publishing, Boston, Massachusetts, USA, pp. 800. 